Pacific

Journal of

Mathematics

ALGEBRAIC STRUCTURE OF QUASIRADIAL SOLUTIONS TO THE $\boldsymbol{\gamma}$-HARMONIC EQUATION

VLADIMIR G. TKACHEV 


\title{
ALGEBRAIC STRUCTURE OF QUASIRADIAL SOLUTIONS TO THE $\gamma$-HARMONIC EQUATION
}

\author{
VLADIMIR G. TKACHEV
}

\begin{abstract}
We obtain an explicit representation for quasiradial $\boldsymbol{\gamma}$-harmonic functions, which shows that these functions have an essentially algebraic nature. We give a complete description of all $\gamma$ that admit algebraic quasiradial solutions. Unlike the cases $\gamma=\infty$ and $\gamma=1$, only finitely many algebraic solutions are shown to exist for any fixed $|\gamma|>1$. A special extremal series of $\boldsymbol{\gamma}$ corresponds exactly to the well known ideal $\boldsymbol{m}$-atomic gas adiabatic constant $\gamma=(2 m+3) /(2 m+1)$.
\end{abstract}

\section{Introduction}

We study specific solutions to the quasilinear equation

$$
u_{x x}\left((\gamma+1) u_{x}^{2}+(\gamma-1) u_{y}^{2}\right)+4 u_{x y} u_{x} u_{y}+u_{y y}\left((\gamma+1) u_{y}^{2}+(\gamma-1) u_{x}^{2}\right)=0
$$

where $|\gamma|>1$ or $\gamma=1$. Let $L_{\gamma}[u]$ denote the left-hand side of this equation. A solution of the form

$$
u(x, y)=\rho^{k} f(\theta), \quad k \geq 1,
$$

where $\rho$ and $\theta$ are the polar coordinates in the $(x, y)$-plane, is said to be quasiradial. The origin of this study goes back to the well-known $p$-Laplace equation

$$
\operatorname{div}\left(|\nabla u|^{p-2} \nabla u\right)=0
$$

which is the divergence form of (1) with $p=2 \gamma /(\gamma-1)$. We call solutions to (1) $\gamma$-harmonic functions.

The existence and integral representations for quasiradial $\gamma$-harmonic functions were established by G. Aronsson [1968; 1984; 1986; 1989; 1992], who also named them. He showed in [Aronsson 1984] (see also [Persson 1989] and [Aronsson

MSC2000: primary 35J15; secondary 31A05, $14 \mathrm{H} 05$.

Keywords: $\gamma$-harmonic function, quasiradial solution, algebraic function.

The author was supported by Russian President grant for young doctorates \#00-15-99274, grant RFBR \# 03-01-00304 and grant of MHE \# E 02-11.0-39. 
1986]) that single-valued quasiradial $\gamma$-harmonic functions exist only for those exponents $k$ in (2) that satisfy the characteristic equation

$$
(2 N-1)(\gamma+1) k^{2}-2\left(N^{2} \gamma+2 N-1\right) k+N^{2}(1+\gamma)=0, \quad N \in \mathbb{N} .
$$

We refer to the corresponding solution as the $N$-solution to (1). One can easily see that 0 -solutions are constants and 1 -solutions are linear functions. We will call such solutions trivial.

The limit case $\gamma=\infty$ reduces to the standard Laplace equation $\Delta u=0$, and the corresponding $N$-solutions are harmonic polynomials of degree $N$. Harmonic polynomials are algebraic functions: they obviously satisfy a polynomial identity $P(x, y, u) \equiv 0$. We show that this property is still valid for $N$-solutions of the Aronsson equation

$$
u_{x x} u_{x}^{2}+2 u_{x y} u_{x} u_{y}+u_{y y} u_{y}^{2}=0
$$

which is the case $\gamma=1$.

In this paper we study this algebraicity property for general values of $\gamma$. To make this point more explicit, we note that for $\gamma \neq \infty$ a (weak) solution of (1) is normally in the class $C^{1, \alpha}$. In particular, quasiradial solutions have a Hölder singularity near the origin, and one should consider them "singular solutions" (the terminology is borrowed from [Aronsson 1989]). This nonregular character is a consequence of the general situation for $\gamma$-harmonic functions near their singular points (points at which $|\nabla u|=0$ ); see [Ural'tseva 1968; Evans 1982; Lewis 1983].

Our key result is an explicit parametric representation for $N$-solutions:

$$
\begin{aligned}
x+i y & =e^{i \phi}\left(\mu \zeta|\zeta|^{2(N-1)}+\bar{\zeta}^{2 N-1}\right), \\
u_{N} & =C|\zeta|^{k(2 N-1)-N} \cdot \operatorname{Re} \zeta^{N} .
\end{aligned}
$$

Here $\zeta \in \mathbb{C}$ is the parametrization variable, $\phi$ is an arbitrary constant, $k=k(N, \gamma)$ is the biggest root of (3), and $\mu$ is defined by (34) below. We show also that (5) represents an entire graph over the $(x, y)$-plane when $\zeta$ runs through the complex plane $\mathbb{C}$.

It follows immediately from (3) and (5) that $u_{N}$ is an algebraic function whenever $k(N, \gamma)$ is a rational number. This makes more explicit the already mentioned Hölder behavior of quasiradial solutions at their singular points.

In contrast with (4), we show that for any rational $|\gamma|>1$ the class of algebraic $N$-solutions is necessarily finite, in the sense that the upper bound

$$
N \leq\left\lfloor\frac{q^{2}\left(p^{2}+2-q^{2}\right)}{2 p^{2}}\right\rfloor
$$


holds, where $\gamma=p / q$ with $q$ and $p$ coprime. (As usual, $\lfloor x\rfloor$ denotes the integer part of $x$.) In particular, this yields the absence of algebraic solutions for all integers $\gamma$ with $|\gamma| \geq 2$.

Denote by $\mathscr{A}$ the set of all $\gamma \in \mathbb{Q},|\gamma|>1$, such that (1) admits nontrivial algebraic $N$-solutions. Then $\gamma \in \mathscr{A}$ if and only if $-\gamma \in \mathscr{A}$ (see Section 4). In Section 5 we show that for all rational $\gamma=p / q \in \mathscr{A}$ with $\gamma>1$, we have the two-sided bound

$$
2+q \leq p \leq q^{2}-2
$$

These inequalities are sharp. Moreover, in Section 6 we prove that the equality $q=p+2$ holds if and only if $q$ is an odd number, $q \geq 3$. This yields the so-called minimal series

$$
\gamma=\frac{2 N+3}{2 N+1}, \quad N=2,3,4, \ldots,
$$

and $N$ is the index of the corresponding algebraic quasiradial solution, which is unique for a given $\gamma$.

We end this introduction with one possible physical interpretation of (6), which gives a motivation of our choice of $\gamma$ instead of $p$. Observe that (1) is a homogeneous form of the gas dynamics equation

$$
L_{\gamma}[\phi]=2 \Delta \phi
$$

for the potential of the gas velocity $\phi$ [Bers 1958, p. 9]. The parameter $\gamma$ in (7) is the adiabatic gas constant, that is, the ratio of the specific heats of the gas at constant volume and constant pressure; see [Milne-Thomson 1960], for example. For all known gas models, the adiabatic constant is found to be $\gamma=(k+1) / k$, where $k$ is the number of degrees of freedom of molecules in the gas. In particular, $\gamma$ is a rational number.

The most important model for applications is that of an ideal $m$-atomic gas; then $\gamma$ is given by

$$
\gamma_{m}=\frac{2 m+3}{2 m+1}, \quad m=1,2, \ldots
$$

The case $m=2$, or $\gamma_{2}=\frac{7}{5}$, describes the earth's atmosphere.

Equation (7) is of nondegenerate elliptic type for all adiabatic exponents $\gamma>1$. It is proved in [Tkachev and Zorina 2005] that for any integer $N \geq 2$ there exists a solution of (7) that is real analytic in all of $\mathbb{R}^{2}$ and has nontrivial polynomial growth $k_{N}>1$ (where $k_{N}$ is defined by (3)).

Now, the quasiradial $N$-solutions to (1) can be naturally regarded as the cones (after a suitable scale renormalization) over the corresponding $N$-solutions to (7). In other words, (1) represents a microscopy level of the gas flow. In this connection, 
the coincidence of the minimal series (6) and the natural adiabatic constants (8) implicitly underlies the algebraic character of the corresponding $N$-solutions. We observe also that in this case we have $N=m$, i.e. the atomic number is equal to the index of the corresponding $N$-solution.

\section{The separation equation}

We start with the basic properties of the wave function $f(\theta)$. For technical reasons, we assume that $|\gamma|>1$. The case $\gamma=1$ requires some more care because the separate equation (12) is degenerate. However, all results formulated below are still valid in this limit case if we suppose that $k>1$.

Separation of variables in (2) yields the ordinary differential equation

$$
\begin{aligned}
f^{\prime \prime}\left((\gamma-1) k^{2} f^{2}+(\gamma+1) f^{\prime 2}\right) & \\
& +\left(f^{\prime 2}(k(\gamma+3)-2)+((1+\gamma) k-2) k^{2} f^{2}\right) f k=0
\end{aligned}
$$

where the prime denotes the derivative with respect to $\theta$. Letting $W=f^{\prime 2}(\theta)$, $Z=f^{2}(\theta)$, we can rewrite this as

$$
\frac{d W}{d Z}=-\frac{k((\gamma+3) k-2) W+k^{3}((\gamma+1) k-2) Z}{(\gamma+1) W+(\gamma-1) k^{2} Z},
$$

which splits into the linear system

$$
\begin{aligned}
W^{\prime}(\xi) & =-k((\gamma+3) k-2) W-k^{3}((\gamma+1) k-2) Z, \\
Z^{\prime}(\xi) & =(\gamma+1) W+(\gamma-1) k^{2} Z .
\end{aligned}
$$

One can easily verify that the general solution to this system is

$$
\begin{aligned}
W(\xi) & =C_{1} k^{2} e^{-2 k^{2} \xi}+C_{2} k((\gamma+1) k-2) e^{2\left(k-k^{2}\right) \xi}, \\
Z(\xi) & =-C_{1} e^{-2 k^{2} \xi}-C_{2}(\gamma+1) e^{2\left(k-k^{2}\right) \xi},
\end{aligned}
$$

where $C_{1}$ and $C_{2}$ are arbitrary constants. Then

$$
W+k^{2} Z=-k \eta^{k-1} C_{2} \quad W+\lambda^{2} Z=\frac{2 k}{\gamma+1} \eta^{k} C_{1},
$$

where $\eta=e^{-2 \xi k}$ and $\lambda^{2}=k^{2}-2 k /(\gamma+1)$. The right-hand side of the latter identity is positive for all $|\gamma|>1$ and $k \geq 1$, so we can define

$$
\lambda:=\sqrt{k^{2}-\frac{2 k}{\gamma+1}} .
$$

Then elimination of $\eta$ in (10) yields $\left(W+k^{2} Z\right)^{k}=C_{3}\left(W+\lambda^{2} Z\right)^{k-1}$. Since (9) is a homogeneous equation, it suffices to study the case $C_{3}=1$. Thus we have the 
first-order differential equation

$$
\left(f^{\prime 2}(\theta)+k^{2} f^{2}(\theta)\right)^{k}=\left(f^{\prime 2}(\theta)+\lambda^{2} f^{2}(\theta)\right)^{k-1} .
$$

We introduce new phase variables $z=f(\theta), w=f^{\prime}(\theta)$, and define the set

$$
\Gamma=\left\{(z, w) \in \mathbb{R}^{2}:\left(w^{2}+k^{2} z^{2}\right)^{k}=\left(w^{2}+\lambda^{2} z^{2}\right)^{k-1}, w^{2}+z^{2} \neq 0\right\} .
$$

The intersection $\Gamma$ with the $z$-axis consists of exactly two points of the form $A^{ \pm}=$ $\left( \pm z_{0}, 0\right)$, called the apexes; we have

$$
z_{0}=\lambda^{k-1} k^{-k}
$$

Lemma 2.1. Let $|\gamma|>1$ and $k>1$. Then $\Gamma$ is a real analytic closed Jordan curve. Moreover, $\Gamma \backslash\left\{A^{+}, A^{-}\right\}$splits into two mutually symmetric graphs having no common points with the z-axis.

Proof. The first statement follows easily from the representation of $\Gamma$ in the polar coordinates $z=r \cos \alpha, w=r \sin \alpha$ :

$$
r=\frac{\left(\sin ^{2} \alpha+\lambda^{2} \cos ^{2} \alpha\right)^{(k-1) / 2}}{\left(\sin ^{2} \alpha+k^{2} \cos ^{2} \alpha\right)^{k / 2}} .
$$

Now, consider $\Gamma$ as the 0 -level set of the function

$$
F(z, w)=\left(w^{2}+k^{2} z^{2}\right)^{k}-\left(w^{2}+\lambda^{2} z^{2}\right)^{k-1} .
$$

We claim that $F_{w}^{\prime} \neq 0$ on $\Gamma \backslash\left\{A^{+}, A^{-}\right\}$. Indeed, suppose $F_{w}^{\prime}\left(z_{1}, w_{1}\right)=0$ and $w_{1} \neq 0$. Then

$$
\frac{1}{2 w_{1}} F_{w}^{\prime}\left(z_{1}, w_{1}\right)=k\left(w_{1}^{2}+k^{2} z_{1}^{2}\right)^{k-1}-(k-1)\left(w_{1}^{2}+\lambda^{2} z_{1}^{2}\right)^{k-2}=0,
$$

which together with $F\left(z_{1}, w_{1}\right)=0$ and $|\gamma|>1$ implies

$$
w_{1}^{2}=\frac{1-\gamma^{2}}{(1+\gamma)^{2}} k^{2} z_{1}^{2} \leq 0
$$

It follows that $w_{1}=z_{1}=0$, which contradicts the definition of $\Gamma$ and implies our claim. Thus, $\Gamma \backslash\left\{A^{+}, A^{-}\right\}$splits into a union of two graphs with respect to the $z$-axis, and the lemma is proved.

Now, we construct a special solution $f(\theta)$ of (12) satisfying the initial condition $f(0)=z_{0}$. With the notation above we have

$$
\frac{d z}{w}=\frac{f^{\prime}(\theta) d \theta}{f^{\prime}(\theta)}=d \theta .
$$


Define

$$
\Theta(\xi)=\int_{A^{+}}^{\xi} \frac{d z}{w}
$$

where $\xi \in \Gamma$ and the integral is taken clockwise along the arc $\left(A^{+}, \xi\right)$ of $\Gamma$. The last integrand a priori has singular behavior when $w$ vanishes (that is, for $\xi=A^{ \pm}$). But it can be shown that these singularities are removable. Indeed, using again the representation of $\Gamma$ as the 0 -level set of function (15), we find

$$
\frac{d z}{w}=-\frac{F_{w}^{\prime} d w}{F_{z}^{\prime} w}=-\frac{k\left(w^{2}+k^{2} z^{2}\right)^{k-1}-(k-1)\left(w^{2}+\lambda^{2} z^{2}\right)^{k-2}}{k^{3}\left(w^{2}+k^{2} z^{2}\right)^{k-1}-(k-1) \lambda^{2}\left(w^{2}+\lambda^{2} z^{2}\right)^{k-2}} \cdot \frac{d w}{z} .
$$

To show that (16) has no singularity it suffices only to verify that the denominator of the right-hand ratio in the preceding equation is nonzero in a neighborhood of the apexes. The corresponding values at $A^{ \pm}$equal $\lambda^{2(k-1)} z_{0}^{2(k-2)}$, which is nonzero. Therefore the integral in (16) is well defined, and it follows that $\Theta(\xi)$ is an analytic function of $\xi$, in the sense that $\Theta(\xi(\tau))$ is analytic for any analytic parametrization $\xi(\tau)$.

Next, observe that $d z / w>0$ within our convention. Applying Lemma 2.1, we conclude that $\Theta(\xi)$ is a strictly increasing function when $\xi$ runs clockwise around $\Gamma$. Define a function $f_{k}(\theta)$ by letting $f_{k}(\Theta(\xi))=z(\xi)$, where $z(\xi)$ is the projection of $\xi$ onto the $z$-axis. Clearly, $f_{k}(\theta)$ becomes a real analytic periodic function, now defined in $\mathbb{R}$. By the symmetry of $\Gamma$, the one-quarter period $T$ satisfies

$$
\frac{T}{4}:=\int_{A^{+}}^{B^{-}} \frac{d z}{w}
$$

where $B^{-}=(0,-1) \in \Gamma$.

Lemma 2.2. $T=2 \pi\left(1-\frac{k-1}{\lambda}\right)$.

Proof. Define a new variable $t$ by letting $w=-z t$. Then, applying (13) we obtain the following parameterization of the $\operatorname{arc} A^{+} B^{-}$:

$$
\begin{gathered}
z(t)=\left(t^{2}+\lambda^{2}\right)^{(k-1) / 2}\left(t^{2}+k^{2}\right)^{-k / 2}, \\
w(t)=-t\left(t^{2}+\lambda^{2}\right)^{(k-1) / 2}\left(t^{2}+k^{2}\right)^{-k / 2} .
\end{gathered}
$$

When $t$ runs between 0 and $+\infty$ the corresponding point $\xi(t)=(z(t), w(t))$ runs clockwise along $A^{+} B^{-}$. Moreover,

$$
d z(t)=t\left(t^{2}+\lambda^{2}\right)^{(k-3) / 2}\left(t^{2}+k^{2}\right)^{-(k+2) / 2}\left(k^{2}(k-1)-k \lambda^{2}-t^{2}\right) d t,
$$

which yields

$$
\frac{d z(t)}{w(t)}=\left(\frac{k}{t^{2}+k^{2}}-\frac{k-1}{t^{2}+\lambda^{2}}\right) d t .
$$


Integration yields

$$
\Theta(z(t), w(t))=\arctan \frac{t}{k}-\frac{k-1}{\lambda} \arctan \frac{t}{\lambda} .
$$

Letting $t \rightarrow+\infty$, we obtain by virtue of (17):

$$
\frac{T}{4}=\frac{\pi}{2}\left(1-\frac{k-1}{\lambda}\right)
$$

the desired equality follows.

From the definition of $\Gamma$ we infer $f_{k}^{\prime}(\Theta(\xi))=w(\xi)$, where $z(\xi)$ is the projection of $\xi$ onto the $z$-axis. In particular,

$$
f_{k}^{\prime}(\theta)=0 \quad \Longleftrightarrow \quad \theta=\frac{T n}{2}, \quad n \in \mathbb{Z} .
$$

Hence, the function $f_{k}(\theta)$ satisfies (12) with the initial data

$$
f_{k}^{\prime}(0)=0, \quad f_{k}(0)=z_{0} \equiv \frac{\lambda^{k-1}}{k^{k}} .
$$

Since (12) is an autonomous system, the general solution of (12) must have the form $f(\theta)=f_{k}(\theta+a)$, where $a$ is an arbitrary constant.

Corollary 2.3. Let $T$ be defined by the equality in Lemma 2.2. For $\theta \in(-T / 4, T / 4)$ we have the following parametrization for $f_{k}(\theta)$ :

$$
\begin{aligned}
f_{k} & =\left(t^{2}+\lambda^{2}\right)^{(k-1) / 2}\left(t^{2}+k^{2}\right)^{-k / 2}, \\
\theta & =\arctan \frac{t}{k}-\frac{k-1}{\lambda} \arctan \frac{t}{\lambda}, \quad t \in \mathbb{R},
\end{aligned}
$$

and for other values of $\theta, f(\theta)$ satisfies the symmetry rules:

$$
f_{k}(\theta)=-f_{k}(T / 2-\theta), \quad f_{k}(-\theta)=f_{k}(\theta) .
$$

Our further objective is to characterize all values of $k>1$ which support the $2 \pi$-periodic wave functions $f_{k}(\theta)$, that is $f_{k}(\theta+2 \pi)=f_{k}(\theta)$. In fact, the latter condition is equivalent to absence of multivalued branches of the corresponding quasiradial solution of (1). The following assertion is a direct consequence of Lemma 2.2.

Proposition 2.4. Let $|\gamma|>1$ and $k \geq 1$. Then $f_{k}(\theta+2 \pi)=f_{k}(\theta)$ if and only if

$$
\frac{k-1}{\lambda}=\frac{N-1}{N}, \quad N \in \mathbb{N},
$$

where $\mathbb{N}$ denotes the set of all positive integers. In this case $f_{k}(\theta)$ is a $2 \pi / N$ periodic function. 
The latter statement is not new. It has appeared in [Aronsson 1984] for $\gamma=1$ and for general $\gamma$ in [Aronsson 1986; Persson 1989]. However, our approach to this result seems to be complementary to those previous works and allows us to arrive at an explicit representation for the quasiradial solutions in the next section.

Proposition 2.5. Let $|\gamma|>1$ and $N \in \mathbb{N}$ be given. Then there exists a unique $k=k(\gamma, N) \geq 1$ such that (9) admits a $2 \pi / N$-periodic solution.

Proof. The trivial case $N=1$ gives $k=1$, by (20). Let $N \geq 2$. Then (20) is equivalent to the quadratic equation

$$
(2 N-1)(\gamma+1) k^{2}-2\left(N^{2} \gamma+2 N-1\right) k+N^{2}(1+\gamma)=0,
$$

which has two separate roots because it has discriminant

$$
4(N-1)^{2}\left(N^{2} \gamma^{2}-2 N+1\right)>4(N-1)^{3}>0 .
$$

Then one can easily infer from the Viète theorem that

$$
\left(k_{1}-1\right)\left(k_{2}-1\right)=-\frac{(N-1)^{2}}{2 N-1} \frac{\gamma-1}{\gamma+1}<0,
$$

where $k_{1} \neq k_{2}$ are the roots of (21). This inequality implies $k_{1}<1<k_{2}$, so that exactly one root $k_{2}>1$ is consistent with our constraint $k>1$.

\section{3. $N$-solutions}

From now on, we adopt a new notation $f_{N}$ for the $N$-th wave function $f_{k}$ with $k=k(\gamma, N)$.

Definition 3.1. Let $N \in \mathbb{N}$. The quasiradial solution of the form

$$
u_{N}(x, y):=C \rho^{k} f_{N}(\theta),
$$

where $C$ is an arbitrary constant, is said to be a basic $N$-solution of (1). Similarly, $u=C \rho^{k} f_{N}(\theta+a)$ with an arbitrary $a \in \mathbb{R}$ is said to be a (general) $N$-solution.

Theorem 3.2. Let $|\gamma|>1, N \in \mathbb{N}$, and $k=k(\gamma, N)$ be the biggest root of (21). Then the basic $N$-solution has the representation

$$
\begin{aligned}
x & =h^{2 N-1}((k+\lambda) \cos \tau+(k-\lambda) \cos (2 N-1) \tau), \\
y & =h^{2 N-1}((k+\lambda) \sin \tau-(k-\lambda) \sin (2 N-1) \tau), \\
u_{N} & =C h^{k(2 N-1)} \cos N \tau,
\end{aligned}
$$

where $\lambda$ is defined by (20), and $\tau \in[0 ; 2 \pi], h>0$ are the parametrization variables. 
Proof. By virtue of (19) we have the following parametrization for $f_{N}(\theta)$ :

$$
\begin{aligned}
f_{N} & =\left(t^{2}+\lambda^{2}\right)^{(k-1) / 2}\left(t^{2}+k^{2}\right)^{-k / 2}, \\
\theta & =\arctan \frac{t}{k}-\frac{k-1}{\lambda} \arctan \frac{t}{\lambda} .
\end{aligned}
$$

Define a new variable $\tau$ by

$$
t=\lambda \tan (N \tau), \quad \tau \in\left(-\frac{\pi}{2 N}, \frac{\pi}{2 N}\right) .
$$

Then we have from (23) and (20)

$$
\theta+(N-1) \tau=\arctan \left(\frac{\lambda}{k} \tan (N \tau)\right)
$$

which by (24) yields

$$
t=k \tan (\theta+(N-1) \tau) .
$$

Inserting (24) and (26) into the first identity in (23), we get

$$
\begin{aligned}
f_{N} & =\left(t^{2}+\lambda^{2}\right)^{(k-1) / 2}\left(t^{2}+k^{2}\right)^{-k / 2} \\
& =z_{0}\left(1+\tan ^{2}(N \tau)\right)^{(k-1) / 2}\left(1+\tan ^{2}(\theta+(N-1) \tau)\right)^{-k / 2} \\
& =z_{0} \cos N \tau\left(\frac{\cos (\theta+(N-1) \tau)}{\cos N \tau}\right)^{k},
\end{aligned}
$$

where $z_{0}$ is defined by (14). On the other hand,

$$
\cos (\theta+(N-1) \tau)=\cos \theta \cos (N-1) \tau(1-\tan (N-1) \tau \tan \theta) .
$$

Now we apply to (28) the addition formula

$$
1-\tan p \tan (\beta-p)=\frac{1}{\cos ^{2} p} \frac{1}{1+\tan \beta \tan p}
$$

with $p=(N-1) \tau, \beta=\arctan (\lambda / k \tan (N \tau))$. From (25), we have $\theta=\beta-p$; therefore

(29) $1-\tan (N-1) \tau \tan \theta$

$$
\begin{aligned}
& =\frac{1}{\cos ^{2}(N-1) \tau} \frac{1}{1+(\lambda / k) \tan (N-1) \tau \tan N \tau} \\
& =\frac{1}{\cos (N-1) \tau} \frac{k \cos N \tau}{k \cos (N-1) \tau \cos N \tau+\lambda \sin (N-1) \tau \sin N \tau} \\
& =\frac{1}{\cos (N-1) \tau} \frac{2 k \cos N \tau}{(k+\lambda) \cos \tau+(k-\lambda) \cos (2 N-1) \tau} .
\end{aligned}
$$


Then, applying (28) and (29) to (27) we obtain

$$
f_{N}=z_{0} \cos N \tau\left(\frac{2 k \cos \theta}{(k+\lambda) \cos \tau+(k-\lambda) \cos (2 N-1) \tau}\right)^{k} .
$$

Taking into account that $u_{N}(x, y)=\rho^{k} f_{N}$ and $x=\rho \cos \theta$, we find

$$
\begin{aligned}
u_{N}(x, y) & =z_{0} \cos N \tau\left(\frac{2 k \cos \theta}{(k+\lambda) \cos \tau+(k-\lambda) \cos (2 N-1) \tau}\right)^{k} \rho^{k} \\
& =(2 k)^{k} z_{0}\left(\frac{x}{(k+\lambda) \cos \tau+(k-\lambda) \cos (2 N-1) \tau}\right)^{k}
\end{aligned}
$$

Setting $h^{2 N-1}$ for the expression in the last brackets, we arrive at

$$
x=h^{2 N-1}((k+\lambda) \cos \tau+(k-\lambda) \cos (2 N-1) \tau),
$$

and $u_{N}=C_{N} h^{k(2 N-1)} \cos N \tau$, where $C_{N}=(2 k)^{k} z_{0}=2^{k} \lambda^{k-1}$.

Finally, to express $y$ we eliminate the polar coordinates as follows

$$
\begin{aligned}
\frac{y}{x} & =\tan \theta=\frac{\lambda \tan N \tau-k \tan (N-1) \tau}{k+\lambda \tan N \tau \tan (N-1) \tau} \\
& =\frac{(k+\lambda) \sin \tau-(k-\lambda) \sin (2 N-1) \tau}{(k+\lambda) \cos \tau+(k-\lambda) \cos (2 N-1) \tau} .
\end{aligned}
$$

Thus we get (22) for all $\tau \in(-\pi / 2 N, \pi / 2 N)$. Using the analyticity of $f_{N}(\theta)$ we conclude that (22) is valid for all $\tau$. The theorem is proved completely.

In order to simplify (22) we make use of a special intermediate parameter $\mu$ :

$$
\mu=\frac{k+\lambda}{k-\lambda},
$$

so $\lambda=k(\mu-1) /(\mu+1)$, and we have from (11)

$$
k=\frac{(1+\mu)^{2}}{2 \mu(\gamma+1)} .
$$

Then, an easy computation shows that (20) becomes

$$
N=\frac{\mu^{2}-1}{2(\mu \gamma-1)} \text {. }
$$

Now we observe that $k>\lambda>0$ for $\gamma>1$, so that $\mu>1$. Similarly, $\gamma<-1$ implies $\mu<-1$. On the other hand, considering (32) as a quadratic equation for $\mu$, namely,

$$
F(\mu):=\mu^{2}-2 \gamma N \mu+(2 N-1)=0,
$$


we find $F(1)=2 N(1-\gamma)$ and $F(-1)=2 N(1+\gamma)$. If $\gamma>1$ then $F(1)<0$, so exactly one root of (33) fits the constraint $\mu>1$; call it

$$
\mu^{+}=N \gamma+\sqrt{N^{2} \gamma^{2}-2 N+1}
$$

Similarly, for $\gamma<-1$ we have

$$
\mu^{-}=N \gamma-\sqrt{N^{2} \gamma^{2}-2 N+1}
$$

Define

$$
\mu \equiv \mu(\gamma, N)= \begin{cases}N \gamma+\sqrt{N^{2} \gamma^{2}-2 N+1} & \text { for } \gamma>1, \\ N \gamma-\sqrt{N^{2} \gamma^{2}-2 N+1} & \text { for } \gamma<-1 .\end{cases}
$$

For later use, note that

$$
\mu(-\gamma, N)=-\mu(\gamma, N) .
$$

By using the homogeneity of (22), one can rewrite it as

$$
\begin{aligned}
x & =h^{2 N-1}(\mu \cos \tau+\cos (2 N-1) \tau), \\
y & =h^{2 N-1}(\mu \sin \tau-\sin (2 N-1) \tau), \\
u_{N} & =C h^{(2 N-1) k} \cos N \tau .
\end{aligned}
$$

A general $N$-solution can be obtained from a certain basic $N$-solution by a suitable rotation in the $(x, y)$ plane. Let

$$
x^{\prime}=x \cos \psi+y \sin \psi, \quad y^{\prime}=-x \sin \psi+y \cos \psi
$$

be such a rotation. Then (36) implies

$$
\begin{aligned}
& x=h^{2 N-1}(\mu \cos \tau+\cos ((2 N-1) \tau+2 N \psi)), \\
& y=h^{2 N-1}(\mu \sin \tau-\sin ((2 N-1) \tau+2 N \psi)), \\
& u \equiv u_{N, \psi}=C h^{(2 N-1) k} \cos N(\tau+\psi) .
\end{aligned}
$$

In particular, $u_{N, 0}=u_{N}$. Thus, (37) gives the representation for general $N$ solutions to (1).

By substituting $X=h \cos \tau$ and $Y=h \sin \tau$ into (37), we obtain an algebraic representation of a basic $N$-solution $u(x, y)$ (see also an equivalent complex form (5) given in the Introduction)

$$
\begin{aligned}
x & =\mu X\left(X^{2}+Y^{2}\right)^{N-1}+\operatorname{Re}(X+i Y)^{2 N-1}, \\
y & =\mu Y\left(X^{2}+Y^{2}\right)^{N-1}-\operatorname{Im}(X+i Y)^{2 N-1}, \\
u_{N} & =C\left(X^{2}+Y^{2}\right)^{(k(2 N-1)-N) / 2} \operatorname{Re}(X+i Y)^{N} .
\end{aligned}
$$


Corollary 3.3. All $N$-solutions are quasialgebraic functions in the sense that $u_{N}^{\alpha}$ is an algebraic function, where

$$
\alpha=\frac{2}{k(2 N-1)-N} .
$$

To illustrate this, we briefly mention a well-known example. (See [Aronsson 1984; Juutinen et al. 2000] for further examples.) Note that $u_{2}=x^{4 / 3}-y^{4 / 3}$ is a basic 2-solution of (4). It is easily verified that $u \equiv u_{2}(x, y)$ satisfies the polynomial identity $27 x^{4} y^{4} u^{3}=\left(x^{4}-y^{4}-u^{3}\right)^{3}$.

\section{Conjugate solutions}

We recall that the main equation (1) can be represented as a $p$-Laplace equation for $p=2 \gamma /(\gamma-1)$. As is well known (see [Aronsson 1992; Aronsson and Lindqvist 1988], for instance), in the two-dimensional case there is the canonical correspondence between $p$-harmonic and $p^{\prime}$-harmonic functions for

$$
\frac{1}{p}+\frac{1}{p^{\prime}}=1 \text {. }
$$

More precisely, given a solution $u$ of $L_{\gamma}[u]=0$ we define the conjugate function $U$ by

$$
U_{x}=|\nabla u|^{2 /(\gamma-1)} u_{y}, \quad U_{y}=-|\nabla u|^{2 /(\gamma-1)} u_{x}, \quad U(0)=0 .
$$

$U(x, y)$ is not necessarily a single-valued function. But at least locally, $U$ is a quasiradial solution of the conjugate equation

$$
L_{-\gamma}[U]=0 .
$$

It turns out that there is a simple relation between conjugate quasiradial solutions. We define an adjoint $N$-solution $u_{N}^{*}$ to $u_{N}$ by setting

$$
\begin{aligned}
x & =h^{2 N-1}(\mu \cos \tau-\cos ((2 N-1) \tau)), \\
y & =h^{2 N-1}(\mu \sin \tau+\sin ((2 N-1) \tau)), \\
u_{N}^{*} & =C h^{(2 N-1) k} \sin N \tau .
\end{aligned}
$$

Applying (37), we obtain an equivalent definition:

$$
u_{N}^{*}=u_{N,-\pi / 2 N} .
$$

We also have $u_{N}^{* *}=-u_{N}$. The functions $u_{N}$ and $u_{N}^{*}$ form a conjugate pair, analogous to conjugate harmonic functions. More precisely, if $\gamma=1$ one can easily derive from the representation above that $u_{N}(x, y)=\operatorname{Re}(x+i y)^{N}$ and $u_{N}^{*}(x, y)=\operatorname{Im}(x+i y)^{N}$. 
Theorem 4.1. Let $u_{N}$ be a basic $N$-solution of (1) and let $U_{N}^{*}$ be an adjoint $N$ solution of (40). Then there is a constant c such that $c u_{N}$ and $U_{N}^{*}$ form a conjugate pair in the sense of (39).

Proof. We can assume that $N \geq 2$. Let $u_{N}$ be an $N$-solution of (1) and let $U$ be the corresponding conjugate function defined by (39), normalized by $U(0)=0$. It follows from the homogeneity of $u_{N}$ that $U$ is a homogeneous function as well. Hence, there is a real $\beta$ such that

$$
U=r^{\beta} G(\theta) .
$$

Here $G(\theta)$ is a priori a multivalued function. We will prove that $U=U_{N}^{*}$ with a suitable constant $C$ in (41).

First, notice that $U$ is a quasiradial solution of (40). Denote by $k$ the growth exponent of $u_{N}$. Then $k>1$ and the components of the gradient $\nabla u_{N}$ are homogeneous functions of order $(k-1)$. Moreover,

$$
\left|\nabla u_{N}\right|^{2}=r^{2 k-2}\left(k^{2} f_{N}^{2}(\theta)+f_{N}^{\prime 2}(\theta)\right) .
$$

In particular, $\nabla u_{N} \neq 0$ for $r \neq 0$. Applying (39) gives

$$
\nabla U=\left[\begin{array}{c}
U_{x}^{\prime} \\
U_{y}^{\prime}
\end{array}\right]=\left|\nabla u_{N}\right|^{2 /(\gamma-1)}\left[\begin{array}{r}
u_{N, y}^{\prime} \\
-u_{N, x}^{\prime}
\end{array}\right]=r^{(k-1)(\gamma+1) /(\gamma-1)}\left[\begin{array}{l}
G_{1}(\theta) \\
G_{2}(\theta)
\end{array}\right],
$$

where the $G_{i}(\theta)$ are certain $2 \pi$-periodic functions of $\theta$. Since $|\gamma|>1$ and $k>1$,

$$
\beta=(k-1) \frac{\gamma+1}{\gamma-1}+1>1 .
$$

At the same time, let $k^{*}$ be the growth exponent of $U_{N}^{*}$. Let $\mu$ and $\mu^{*}$ be the corresponding auxiliary parameters defined by (30) for $k$ and $k^{*}$ respectively. Then it follows from (35) that $\mu^{*}=-\mu$, and from (31) we have

$$
k=\frac{(1+\mu)^{2}}{2(1+\gamma) \mu} \quad \text { and } \quad k^{*}=\frac{\left(1+\mu^{*}\right)^{2}}{2\left(1+\gamma^{*}\right) \mu^{*}}=\frac{(1-\mu)^{2}}{2(\gamma-1) \mu},
$$

where $\gamma^{*}=-\gamma$. Hence

$$
k(1+\gamma)+k^{*}(1-\gamma) \equiv k(1+\gamma)+k^{*}\left(1+\gamma^{*}\right)=2 .
$$

Thus, by (43) we have

$$
\begin{aligned}
\beta & =(k-1) \frac{\gamma+1}{\gamma-1}+1=\frac{k(\gamma+1)}{\gamma-1}-\frac{\gamma+1}{\gamma-1}+1 \\
& =\frac{2-k^{*}(-\gamma+1)}{\gamma-1}-\frac{2}{\gamma-1}=k^{*} .
\end{aligned}
$$


The latter means that $U$ is an $N$-solution of (40) with $\beta=k^{*}$ (in particular, the function $G$ in (42) is single-valued).

It remains only to show that $U$ is an adjoint $N$-solution. Since $U$ is an $N$ solution, there is $\psi$ such that $U=U_{N, \psi}$ in representation (37). Thus, we have to prove that $N \psi= \pm \pi / 2 \bmod \pi$.

Choosing $\tau_{0}=-\psi+\pi / 2 N$ and $h=1$ in the representation (37) for $U=U_{N, \psi}$, we obtain the corresponding Cartesian coordinates $\left(x_{0}, y_{0}\right) \neq 0$. Then $U\left(x_{0}, y_{0}\right)=0$ and applying the Euler theorem on homogeneous functions yields

$$
x_{0} U^{\prime}{ }_{x}\left(x_{0}, y_{0}\right)+y_{0} \frac{\partial U}{\partial y}\left(x_{0}, y_{0}\right)=k^{*} U\left(x_{0}, y_{0}\right)=0 .
$$

Hence, the gradient $\nabla U\left(x_{0}, y_{0}\right)$ is orthogonal to the radius vector $\nabla r\left(x_{0}, y_{0}\right)$ of the point $\left(x_{0}, y_{0}\right)$. On the other hand, one can readily see from (37) that

$$
x_{0}=\left(\mu^{*}-1\right) \cos \left(\frac{\pi}{2 N}-\psi\right), \quad y_{0}=\left(\mu^{*}-1\right) \sin \left(\frac{\pi}{2 N}-\psi\right) .
$$

Hence the polar angle of the point $\left(x_{0}, y_{0}\right)$ satisfies

$$
\theta_{0} \equiv \frac{\pi}{2 N}-\psi \quad \bmod \pi
$$

By (39), the gradients of $u_{N}$ and $U$ are mutually orthogonal. From (44) we infer that the vectors $\nabla u_{N}\left(x_{0}, y_{0}\right)$ and $\nabla r\left(x_{0}, y_{0}\right)$ are collinear. Since

$$
\nabla u_{N}=f_{N}(\theta) r^{k-1} \nabla r+r^{k} f_{N}^{\prime}(\theta) \nabla \theta \quad \text { and } \quad\langle\nabla \theta ; \nabla r\rangle=0,
$$

we conclude that $f_{N}^{\prime}\left(\theta_{0}\right)=0$. Hence, by (18) there is $n \in \mathbb{Z}$ such that

$$
\theta_{0}=\frac{T n}{2}=\frac{\pi n}{N}
$$

which by (45) yields $N \psi \equiv \pi / 2 \bmod \pi$, and the theorem follows.

\section{Algebraic $N$-solutions}

In this section we settle the following question: For which rational numbers $\gamma \in \mathbb{Q}$ such that $|\gamma|>1$ does Equation (1) admit nontrivial algebraic solutions? (Recall that nontrivial means $N \geq 2$.) It follows from (37) that a general $N$-solution is algebraic if and only if basic $N$-solutions are. Therefore, in what follows it suffices to consider basic $N$-solutions.

Lemma 5.1. Let $\gamma \in \mathbb{Q},|\gamma|>1$ and $N \in \mathbb{N}$. Then inclusions $k(\gamma, N) \in \mathbb{Q}$, $\lambda(\gamma, N) \in \mathbb{Q}$ and $\mu(\gamma, N) \in \mathbb{Q}$ are pairwise equivalent.

Proof. It immediately follows from (20) that inclusions $k \in \mathbb{Q}$ and $\lambda \in \mathbb{Q}$ are equivalent. By (31), $\mu \in \mathbb{Q}$ implies both $k \in \mathbb{Q}$ and $\lambda \in \mathbb{Q}$. On the other hand, if $k \in \mathbb{Q}$ then $\lambda \in \mathbb{Q}$, so by (30) $\mu \in \mathbb{Q}$ and the lemma is proved. 
For convenience, we put $\gamma \in \mathscr{A}$ if there exists an integer $N \geq 2 \operatorname{such}$ that $u_{N}(x, y)$ is an algebraic function. In this case we also define

$$
\mathcal{N}(\gamma)=\left\{N \in \mathbb{N}: u_{N} \text { is an algebraic function }\right\} .
$$

Lemma 5.2. Consider $N \geq 2$ and $\gamma \in \mathscr{A}$ such that $|\gamma|>1$. Then $N \in \mathcal{N}(\gamma)$ if and only if the corresponding exponent $k(\gamma, N)$ is rational.

Proof. Suppose $N \in \mathcal{N}(\gamma)$. Then it follows from (37) and $|\mu|>2 N-1 \geq 3$ that

$$
\left(x^{2}+y^{2}\right)=h^{4 N-2}\left[\mu^{2}+1+2 \mu \cos (2 N \tau+2 \phi)\right] \sim h^{4 N-2} \quad \text { as } h \rightarrow \infty,
$$

while

$$
u_{n}(x, y)=h^{(2 N-1) k} \cos (2 N \tau+\phi),
$$

where $k=k(\gamma, N)$. Thus the growth exponent of $u$ is $k$, and it follows that $k \in \mathbb{Q}$.

Now suppose $k(\gamma, N) \in \mathbb{Q}$. Then (38) gives a rational parametrization of $u_{N}^{2 d}$, where $d$ is the denominator of $k$. Hence $u_{N}(x, y)$ is an algebraic function.

Corollary 5.3. For $\gamma=1$ all $N$-solutions are algebraic functions. Thus $\mathcal{N}(1)=\mathbb{N}$.

The next assertion is an easy corollary of (43).

Lemma 5.4. Let $\gamma \in \mathbb{Q}$. Then $k_{N}$ is rational if and only if $k_{N}^{*}$. In particular,

$$
\mathcal{N}(\gamma)=\mathcal{N}(-\gamma) .
$$

By Lemma 5.4, we can assume without loss of generality that $\gamma>1$. In what follows, we suppose that $p$ and $q$ have no common divisors. By Lemma 5.2, $\gamma$ is in $\mathscr{A}$ if and only if there is an integer $N \geq 2$ such that $k(\gamma, N)$ is rational. By Lemma 5.1, this is equivalent to the existence of a rational solution $\mu=A / B>1$ of (32):

$$
N=\frac{q\left(A^{2}-B^{2}\right)}{2 B(A p-B q)}, \quad A>B .
$$

Thus, we arrive at the diophantine equation

$$
A^{2} q-2 A B p N+q(2 N-1) B^{2}=0 .
$$

Theorem 5.5. The following assertions are equivalent:

(i) $\gamma=p / q \in \mathscr{A}$, with $N \in \mathcal{N}(\gamma)$ and $N \geq 2$.

(ii) Equation (46) has an integer solution $(A, B)$, with $A, B \in \mathbb{Z}$ and $A>B \geq 1$.

(iii) The discriminant

$$
N^{2} p^{2}-q^{2}(2 N-1)
$$

is a squared integer. 
Moreover, if $\gamma \in \mathscr{A}$ with $\gamma \neq 1$, the set $\mathcal{N}(\gamma)$ is finite and the upper bound

$$
N<\frac{q^{2}\left(p^{2}+2-q^{2}\right)}{2 p^{2}} .
$$

holds. In particular, $q \geq 3$ and

$$
q+1 \leq p \leq q^{2}-1 .
$$

Proof. Clearly, we have only to establish the equivalence (ii) and (iii), and the only nontrivial implication is (iii) $\Rightarrow$ (ii).

Let (iii) be true. Then $p=q \gamma>q$ and the discriminant equals $d^{2}$ for some $d \in \mathbb{N}$. Since

$$
N^{2} p^{2}-q^{2}(2 N-1)=N^{2}\left(p^{2}-q^{2}\right)+q^{2}(N-1)^{2}>0,
$$

we have $d>0$. Set $V=A / B$ and consider the quadratic equation

$$
F(V):=V^{2} q-2 p N V+q(2 N-1)=0
$$

associated with (46). This equation has two distinct rational solutions $v_{1}$ and $v_{2}$, with $v_{1}<v_{2}$. Since

$$
F(2 N-1)=-2(N-1)(2 N-1)(p-q)<0,
$$

we have $v_{2}>2 N-1$. Set $v_{2}=A / B$, where $A$ and $B$ have no common divisors and $B>0$. Hence, $A>(2 N-1) B>B$ and $(A, B)$ is a desired solution of (ii).

To establish the finiteness of $\mathcal{N}(\gamma)$, we write

$$
d^{2}=\left(N p-\frac{q^{2}}{p}\right)^{2}+\frac{q^{2}\left(p^{2}-q^{2}\right)}{p^{2}} .
$$

Then (49) yields

$$
d>N p-\frac{q^{2}}{p},
$$

while the Bernoulli inequality and (49) imply the upper bound

$$
d<\left(N p-\frac{q^{2}}{p}\right)+\frac{q^{2}\left(p^{2}-q^{2}\right)}{2 p\left(N p^{2}-q^{2}\right)} .
$$

Since $p$ and $q$ have no common divisors, we can write

$$
\frac{q^{2}}{p}=M+\frac{m}{p}
$$

where $M>0$ is an integer and $m \in\{1,2, \ldots, p-1\}$. On the other hand, since $m=q^{2}-M p$, it follows that $m$ and $p$ have no common divisors. Using $N p \in \mathbb{N}$ 
and the strict inequalities (50) and (51), we then obtain

$$
\frac{q^{2}\left(p^{2}-q^{2}\right)}{2 p\left(N p^{2}-q^{2}\right)}>\frac{1}{p},
$$

which easily implies (47).

To verify that $q \geq 3$ we notice that the cases $q=1$ and $q=2$ together with (47) easily yield the contradiction $N<2$.

The first inequality in (48) immediately follows from $p>q$. Finally, to prove the second, note that $d^{2}<N^{2} p^{2}$, whence $d<N p$. Taking into account that $d$ is an integer we obtain $d \leq N p-1$, or, what is the same, $0 \leq(N p-1)^{2}-d^{2}=$ $2 N\left(q^{2}-p\right)+1-q^{2}$. Hence

$$
q^{2}-p \geq\left(q^{2}-1\right) / 2 N \geq 0 .
$$

Therefore $p<q^{2}$, and since $q$ and $p$ are integers we arrive at the stronger inequality $q+1 \leq p \leq q^{2}-1$, completing the proof of the theorem.

Corollary 5.6. If $\gamma$ is an integer with $|\gamma|>1$, then (1) has no nontrivial algebraic $N$-solutions.

Proposition 5.7. $\mathscr{A}=\left\{\frac{2 N-1+s^{2}}{2 s N}: s \in \mathbb{Q} \cap(0,1), N \in \mathbb{N}\right\}$.

Proof. We must show that the right-hand side describes the values of $\gamma>1$ such that the discriminant $N^{2} p^{2}-q^{2}(2 N-1)$ is a squared integer. We do this using the standard rationalization technique for Pell-type equations: the condition on the discriminant is equivalent to the existence of a positive integer-valued solution $(x, y)$ of

$$
N^{2} x^{2}-(2 N-1) y^{2}=1
$$

with $x>y$. Assuming $(x, y)$ is such a solution, write $x=(1+s y) / N$, with $s$ necessarily rational. Substituting this equality in (53) gives

$$
y=\frac{2 s}{2 N-1-s^{2}}, \quad x=\frac{2 N-1+s^{2}}{N\left(2 N-1-s^{2}\right)} .
$$

Now

$$
\gamma=\frac{p}{q}=\frac{x}{y}=\frac{2 N-1+s^{2}}{2 s N}
$$

as claimed; moreover

$$
\gamma-1=\frac{(s-2 N+1)(s-1)}{2 s N},
$$

so $\gamma>1$ is equivalent to $s \in(0,1) \cup(2 N-1,+\infty)$. Since the expression for $\gamma$ is invariant under the involution $s \mapsto(2 N-1) / s$, it suffices to take $s \in(0,1)$. 


\section{Maximal and minimal series}

Now we study how $\mathscr{A}$ depends on the fractional decomposition $\gamma=p / q$. We suppose as before that $p$ and $q$ have no common divisors.

Proposition 6.1 (Maximal series). For $p / q \in \mathscr{A}$ we have the sharp bound

$$
p \leq q^{2}-2
$$

with equality only for odd denominator $q=2 s+1, p=4\left(s^{2}+s\right)-1$, and $N=$ $s(s+1)$, where $s$ is a positive integer. If the denominator $q=2 s$ is even, we have a stronger inequality

$$
p \leq \frac{q^{2}-2}{2}=2 s^{2}-1
$$

with equality if and only if

$$
p=\frac{q^{2}-2}{2}, \quad N=\frac{q^{2}-4}{4} .
$$

Proof. First we prove (54). In view of (48), it suffices to exclude the case $q=p^{2}-1$. Assume the contrary. Then (52) and (47) yield

$$
q^{2} \leq 2 N<q^{2} \frac{\left(p^{2}+2-q^{2}\right)}{p^{2}},
$$

which easily implies $q^{2} \leq 2$, contradicting the lower bound $q \geq 3$ of Theorem 5.5.

In order to analyze the equality case, assume that $p=q^{2}-2$. It follows from (52) that $4 N \geq q^{2}-1=p+1$. The discriminant can be rewritten as

$$
d^{2}=N^{2} p^{2}-(2 N-1)(p+2)=(N p-1)^{2}-(4 N-1-p) \leq(N p-1)^{2},
$$

where $d>0$ is an integer. Hence $d \leq(N p-1)$.

On the other hand,

$$
d^{2}=(N p-2)^{2}+(p-2)(2 N-1)>(N p-2)^{2},
$$

which together with the preceding inequality implies $d=N p-1$, and consequently

$$
p=q^{2}-2=4 N-1 \text {. }
$$

In particular, $q$ must be odd. One readily sees that in this case $N$ is an integer. Thus, the first case of the corollary is proved.

To prove the second statement we suppose $q=2 s$. Theorem 5.5 says that $s \geq 2$. Since $p / q$ is irreducible, $p$ is odd.

By Theorem 5.5 the discriminant satisfies

$$
d^{2}=N^{2} p^{2}-4 s^{2}(2 N-1) .
$$


The last identity shows that $d$ has the same parity as $N p$. Thus $d \leq p N-2$. At the same time,

$$
(N p-2)^{2} \geq d^{2}=(N p-2)^{2}+4\left(N p-1-s^{2}(2 N-1)\right),
$$

so $p-2 s^{2} \leq-\left(s^{2}-1\right) / N$. This inequality, together with $s \geq 2$, yields

$$
p<2 s^{2}=\frac{q^{2}}{2} .
$$

Inequality (55) follows since $q$ is even.

To analyze the equality case in (55) we observe that $d>N p-3$; otherwise we would have

$$
(N p-3)^{2} \leq d^{2}=N^{2} p^{2}-2(2 N-1)(p+1),
$$

which implies $2 N(p-2) \leq 7-2 p$, leading to a contradiction. Thus $d=N p-2$ and a straightforward computation shows that $N=(p-1) / 2=s^{2}-1$, which completes the proof.

Proposition 6.2 (Minimal series). For $p / q \in \mathscr{A}$ we have the lower bound

$$
p \geq q+2,
$$

with equality if and only if $q=2 s+1$ is odd, $p=q+2$ and $N=(q-1) / 2$.

Proof. From (48) we have $p \geq q+1$. Again, we argue by contradiction and assume that $p=q+1$. Then the discriminant is of the form

$$
N^{2}(q+1)^{2}-q^{2}(2 N-1)=d^{2},
$$

with $d$ a positive integer. Hence

$$
d^{2}=q^{2}(N-1)^{2}+2 q N^{2}+N^{2}=\left(q(N-1)+\frac{N^{2}}{N-1}\right)^{2}-\frac{(2 N-1) N^{2}}{(N-1)^{2}} .
$$

In particular,

$$
d<q(N-1)+\frac{N^{2}}{N-1}=q(N-1)+N+1+\frac{1}{N-1},
$$

which implies

$$
d \leq q(N-1)+N+1 \equiv d_{1}+1 .
$$

On the other hand,

$$
d_{1}^{2}=(q(N-1)+N)^{2}=q^{2}(N-1)^{2}+2 q N(N-1)+N^{2}<d^{2},
$$

so $d=d_{1}+1$. By virtue of (57) we get $2 q=2 N-1$, again leading to a contradiction. Thus $p \geq q+2$ and (56) is proved. 
Now assume that $p=q+2$. Arguing as above we obtain

$$
d<(N-1) q+2 N+2+\frac{2}{N-1} \leq(N-1) q+2 N+3 .
$$

At the same time,

$$
d^{2} \equiv q^{2}(N-1)^{2}+4 q N^{2}+4 N^{2}>((N-1) q+2 N)^{2},
$$

which implies by the strong inequality in (58) that

$$
(N-1) q+2 N+1 \leq d \leq(N-1) q+2 N+2 .
$$

But it follows from (59) that $d$ and $(N-1) q$ have the same parity. This gives exactly one choice in the last inequality,

$$
d=(N-1) q+2 N+2,
$$

which after comparison with the definition of $d$ in (57) implies $q=2 N+1$. The required relation follows.

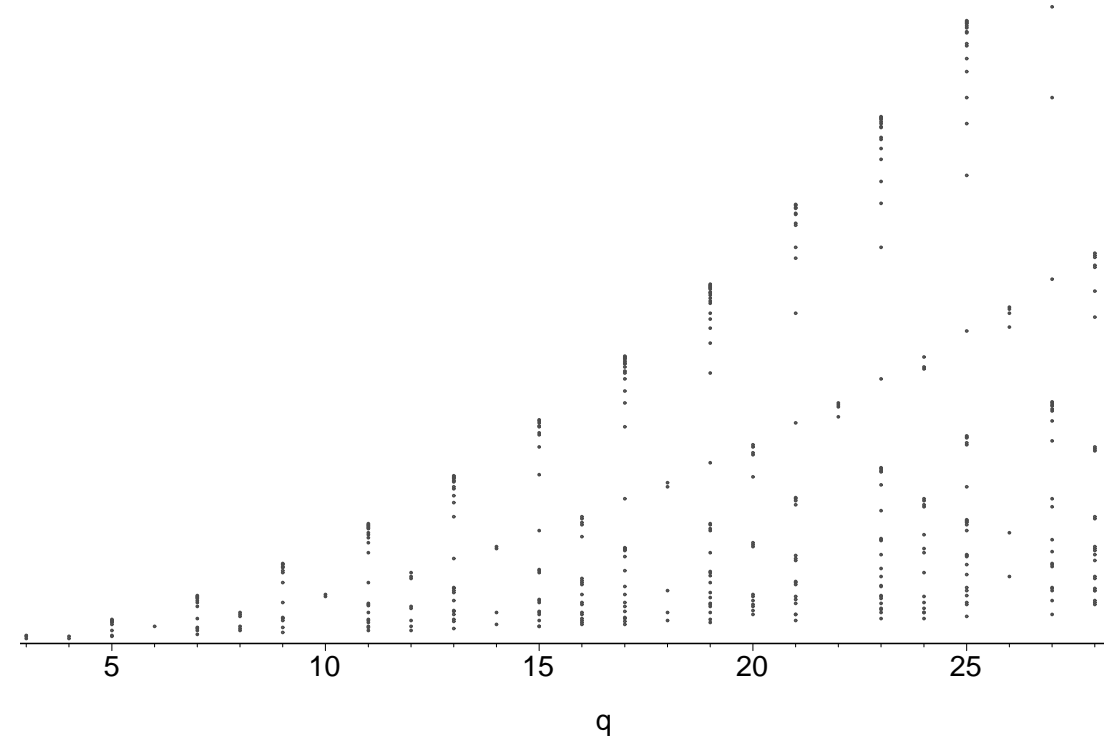

Figure 1. "Algebraic" constants $p / q=\gamma \in \mathscr{A}$ for small denominators $q \leq 30$. 


\section{Concluding remarks}

Our formula (5) can also be deduced by using the general representation of $p$ harmonic functions in the plane near their singular points. This is established in [Iwaniec and Manfredi 1989] and [Manfredi 1991] by using the hodograph method. Our approach, nevertheless, is more direct and does not use the quasiregularity of the complex gradient of the $N$-solutions.

\section{Acknowledgment}

The author thanks the anonymous reviewer for much constructive advice and for the reference to [Iwaniec and Manfredi 1989] and [Manfredi 1991]. He also thanks the editor, Dr. Silvio Levy, for many helpful comments and important suggestions that led to considerable improvements in this paper.

\section{References}

[Aronsson 1968] G. Aronsson, "On the partial differential equation $u_{x}^{2} u_{x x}+2 u_{x} u_{y} u_{x y}+u_{y}^{2} u_{y y}=0$ ", Ark. Mat. 7 (1968), 395-425. MR 38 \#6239 Zbl 0162.42201

[Aronsson 1984] G. Aronsson, "On certain singular solutions of the partial differential equation $u_{x}^{2} u_{x x}+2 u_{x} u_{y} u_{x y}+u_{y}^{2} u_{y y}=0$ ", Manuscripta Math. 47:1-3 (1984), 133-151. MR 85m:35011 $\mathrm{Zbl} 0551.35018$

[Aronsson 1986] G. Aronsson, "Construction of singular solutions to the $p$-harmonic equation and its limit equation for $p=\infty$ ", Manuscripta Math. 56:2 (1986), 135-158. MR 87j:35070 Zbl 0594.35039

[Aronsson 1989] G. Aronsson, "Representation of a $p$-harmonic function near a critical point in the plane”, Manuscripta Math. 66:1 (1989), 73-95. MR 91c:31009 Zbl 0702.35049

[Aronsson 1992] G. Aronsson, "Aspects of p-harmonic functions in the plane", pp. 9-34 in Summer School in Potential Theory (Joensuu, 1990), edited by I. Laine, Joensuun Yliop. Luonnont. Julk. 26, Univ. Joensuu, Finland, 1992. Preprint: Linköpings Universitet LiTH-MAT-R-91-38, Sweden, 1991. MR 93j:35069 Zbl 0756.31007

[Aronsson and Lindqvist 1988] G. Aronsson and P. Lindqvist, "On $p$-harmonic functions in the plane and their stream functions", J. Differential Equations 74:1 (1988), 157-178. MR 90g:31003 Zbl 0659.35037

[Bers 1958] L. Bers, Mathematical aspects of subsonic and transonic gas dynamics, Surveys in Applied Math. 3, Wiley, New York, 1958. MR 20 \#2960 Zbl 0083.20501

[Evans 1982] L. C. Evans, "A new proof of local $C^{1, \alpha}$ regularity for solutions of certain degenerate elliptic p.d.e", J. Differential Equations 45:3 (1982), 356-373. MR 84a:35082 Zbl 0508.35036

[Iwaniec and Manfredi 1989] T. Iwaniec and J. J. Manfredi, "Regularity of p-harmonic functions on the plane", Rev. Mat. Iberoamericana 5:1-2 (1989), 1-19. MR 91i:35071 Zbl 0805.31003

[Juutinen et al. 2000] P. Juutinen, P. Lindqvist, and J. J. Manfredi, “The infinity laplacian: examples and observations”, preprint 26, Institut Mittag-Leffler, Djursholm, 2000.

[Lewis 1983] J. L. Lewis, "Regularity of the derivatives of solutions to certain degenerate elliptic equations”, Indiana Univ. Math. J. 32:6 (1983), 849-858. MR 84m:35048 Zbl 0554.35048 
[Manfredi 1991] J. J. Manfredi, "Isolated singularities of $p$-harmonic functions in the plane", SIAM J. Math. Anal. 22:2 (1991), 424-439. MR 92c:31016 Zbl 0736.35037

[Milne-Thomson 1960] L. M. Milne-Thomson, Theoretical hydrodynamics, 4th ed., Macmillan, New York, 1960. Reprinted by Dover, New York, 1973. MR 22 \#3286 Zbl 0089.42601

[Persson 1989] L. Persson, Quasiradial solutions of the p-harmonic equation in the plane and their stream functions, Licentiate Thesis, Luleå Tekniska Universitet, Sweden, 1989.

[Tkachev and Zorina 2005] V. G. Tkachev and I. A. Zorina, "Entire solutions of the Simon equation", pp. 55-74 in Proceedings of conference on geometrical analysis and applications (Volgograd, 2004), edited by A. G. Losev, Volgograd State Univ., 2005. In Russian.

[Ural'tseva 1968] N. N. Ural'tseva, "Degenerate quasilinear elliptic systems”, Zap. Naučn. Sem. Leningrad. Otdel. Mat. Inst. Steklov. (LOMI) 7 (1968), 184-222. In Russian. MR 39 \#5942 Zbl 0199.42502

Received October 16, 2004. Revised January 28, 2005.

VLADIMIR G. TKACHEV

MATHEMATICAL DEPARTMENT

Volgograd State UNIVERSITY

2-JA PRODOLNAJA 30 Volgograd

400062 RUSSIA

vladimir.tkachev@volsu.ru

http://www.math.kth.se/ tkatchev 